\title{
Practices for Capturing Short Important Thoughts
}

\author{
Gillian Hayes, Jeffrey S. Pierce, and Gregory D. Abowd \\ GVU Center \& College of Computing \\ Georgia Institute of Technology \\ Atlanta, GA 30332-0280 USA \\ \{gillian,jpierce, abowd\}@cc.gatech.edu

\section{RESULTS AND IMPLICATIONS}

\begin{abstract}
In this paper we describe a user study designed to understand current practices for recording and utilizing information in everyday life. We describe a subset of the results that suggest that improving on current practices will require physical and digital artifacts, flexibility, multimodality, and ubiquity.
\end{abstract}

\section{Keywords}

Informal note taking, ubiquitous computing

\section{INTRODUCTION}

Despite advances in personal information management devices, users increasingly have difficulty documenting, prioritizing, and organizing daily tasks, not to mention remembering all the details of daily life[2]. A ubiquitous system for recording and accessing notes, ideas, lists, and other information could help off-load users by augmenting their memory. As a first step toward understanding how to design such a system, we queried users about their current practices and unfulfilled needs.

We studied how users currently record, organize, and access information. Our goal was to identify core behaviors that a new system would need to support, difficulties that users currently encounter, and actions that the system might perform automatically with minimal user intervention

\section{METHOD}

We surveyed potential users by means of interviews and questionnaires. We interviewed a small set of participants to gather initial data and refine our questions. We then distributed the questionnaire and analyzed the results. Because some interesting questions arose from that analysis, we conducted follow-up interviews with a subset of the original interview and questionnaire participants.

We worked with a total sample size of 22 , including 15 males and 7 females ranging in age from 22 to 64 . The participant group included full time students as well as consultants, sales professionals, attorneys, educators, engineers, accountants, and those in the service industries. Participation in the study was strictly voluntary.

Copyright is held by the author/owner(s).

CHI 2003, April 5-10, 2003, Ft. Lauderdale, Florida, USA.

ACM 1-58113-637-4/03/0004
Our analysis of the survey data suggests that a nextgeneration system should provide physical and digital artifacts, flexibility, multi-modality, and ubiquity. In addition, a system with a mixed-initiative interface [3]could provide useful advanced functionality.

\section{The Importance of Physical and Digital Artifacts}

People want the conveniences of both physical and digital artifacts. When asked which mechanisms participants "use for jotting down notes, ideas, or to-do items", $68 \%$ of the mechanisms cited by users included handwritten input on a variety of physical artifacts, including notepads, calendars, and Post-It trademarked. 11 out of the 12 participants who owned a PDA, for example, also mentioned using handwritten mechanisms such as scrap paper and notebooks.

While traditional writing is appealing as an input method, users also wanted the benefits of information in digital form, including increased longevity and the ability to reorganize information. Of the participants with both digital and analogue methods available, $44 \%$ reported copying information from handwritten to digital format. Other responses expanded further on the desire for digitization and the ability to transfer between digital and analogue modes. Some users referred to the problems of carrying too much paper as well as the ease with which their paper notes could be lost or destroyed. When we asked participants to "list three things you'd like to improve about your current note-taking process," the most frequently mentioned items were better organization (12) and digitization (7). These results suggest that a future system should provide synchronous, background-aware, pen-and-paper input to a digital repository.

\section{Flexibility}

The most often cited uses of recorded information included day planning (35\%), tracking tasks $(26 \%)$, and storing important dates and numbers (12\%), all of which require a great deal of organization and prioritization. Participants indicated a wide variety of methods used for organizing the information they recorded, including chronologically, alphabetically, and by priority. Participants also listed transcription into different forms as a way of organizing their information. For example, some participants used only handwritten formats but copied smaller pieces of paper onto larger ones, once the information had reached a certain age, and then organized these notebooks by filing 
them using a personal system. For many, the organization method also changed based upon the age and type of information being stored. This variety suggests that systems with a single, monolithic approach to organizing information may be more of a hindrance than a help.

\section{Multi-Modality}

In addition to handwritten input, users reported using electronic devices, such as computers and PDAs (23\%), and audio (9\%). Users also convert information between formats. When asked how often they "copy or transcribe recorded information from one form to another, and what kind of copying [they] do," $73 \%$ of participants reported regularly transcribing information from one format to another. These results suggest that a new system must have the ability to receive input and produce output in whichever modality is most appropriate for the situation regardless of the format used for storage.

\section{Ubiquity}

A multi-modal ubiquitous interface could also extend use of the system to environments in which the data may be ubiquitously available but the appropriate interface to the system is not. We identified three types of environments that require better support for recording information: social contexts, traveling, and challenging physical environments. A socially inappropriate context can be an event (movies, concerts, etc) or a one-on-one conversation in which it would be considered rude. Traveling includes any time that the motion of the user requires so much attention that distraction could be dangerous. Challenging physical environments are those in which recording information requires overcoming too many obstacles to seem worthwhile (i.e. bed, shower, surprise meetings).

Many participants noted not only the need for appropriate ubiquitous interfaces but also for constant availability of all of their information. They were often frustrated because what they needed was in a different location. Keeping all of the information in a constantly accessible storage area, either a single repository or networked collection, alleviates this frustration. Furthermore, utilizing a single repository that is always with the user reduces the user's dependence on network connectivity.

\section{Mixed Initiative}

Results suggest that a new system should be adaptive, employing a mixed-initiative interface allowing the user to initiate and/or control system actions as well as respond to system-initiated and controlled activities. Because $73 \%$ of the participants reported transcribing information, any new technology should support data transformation. Users often converted information from one format to another to make it more usable. For example, several participants cited using audio input but were frustrated by the need to transcribe that input to text to make it easier to access and use. Transcription of audio to a more usable format could be performed by the system, removing that burden from the user.

However, currently available technologies for automatic conversion between formats are inadequate given the highly personal nature of the record. Participants reported using individual short-hand and abbreviations, making handwritten to digital transcription difficult in the general case. Audio transcription would be hindered by individual speech nuances.

\section{FURTHER WORK}

Based on the design implications previously discussed, we are building a new system for ubiquitously recording important information using an Intel Personal Server[4] as the central information repository. The Personal Server presents an opportunity to develop a system that will make it possible to store, organize, and access almost all (if not all) of the information that people wish to record throughout an average day. We plan to use Bluetooth ${ }^{\mathrm{TM}}[1]$ to allow users to dynamically incorporate different input and output devices to facilitate the most appropriate capture and access mechanism for any given situation. We plan to support integration with pen-based systems to augment current hand-written methods, tablet-based systems, PDAs, and audio headsets.

\section{CONCLUSIONS}

We studied the information recording practices of 22 participants using questionnaires and interviews. Based on our analysis of the results, we believe that a nextgeneration system should provide pen-and-paper input, flexibility, multi-modality, and ubiquity. We believe that a mixed-initiative system could provide useful advanced functionality. We are now prototyping with an Intel Personal Server that leverages these implications.

\section{REFERENCES}

1. Specification of the Bluetooth System, Bluetooth Special Interest Group, 2001.

2. Blanford, A.E. and Green, T.R.G. Group and Individual Time Management Tools: What You Get is Not What You Need. Personal and Ubiquitous Computing, 5 (4). 213-230.

3. Horvitz, E., Principles of Mixed-Initiative User Interfaces. in Conference on Human Factors in Computing Systems, (New York, NY, 1999), ACM Press.

4. Want, R., Pering, T., Danneels, G. and Kumar, M., The Personal Server: Changing the Way We Think About Ubiquitous Computing. in Ubicomp 2002: Ubiquitous Computing, (Goteberg, Sweden, 2002), Springer-Verlag. 